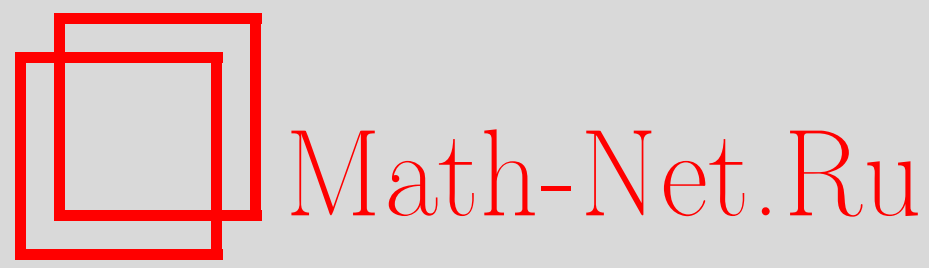

Р. Ф. Бикбаев, О невырожденности амплитудночастотной модуляции для конечнозонных решений интегрируемых нелинейных уравнений, $M a-$ тем. заметки, 1996, том 59, выпуск 1, 53-61

DOI: https://doi.org/10.4213/mzm1693

Использование Общероссийского математического портала MathNet.Ru подразумевает, что вы прочитали и согласны с пользовательским соглашением

http://www.mathnet.ru/rus/agreement

Параметры загрузки:

IP : 52.87 .193 .239

26 апреля 2023 г., 11:57:59

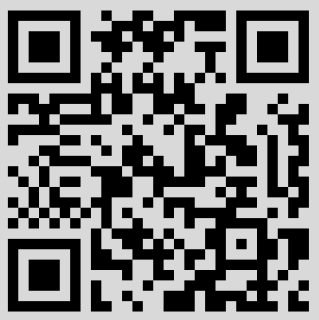




\section{О НЕВЫРОЖДЕННОСТИ АМПЛИТУДНО-ЧАСТОТНОЙ МОДУЛЯЦИИ ДЛЯ КОНЕЧНОЗОННЫХ РЕШЕНИЙ ИНТЕГРИРУЕМЫХ НЕЛИНЕЙНЫХ УРАВНЕНИЙ} Р. Ф. Бикбаев

\section{Введение}

В работах [1] и [2] была доказана важная для приложений теорема об “амплитудно-частотной" модуляции для конечнозонных решений уравнения Кортевега-де Фриза (КдФ). Эта модель является простейшей, поскольку точки ветвления $\lambda_{i}$ соответствующей гиперэллиптической спектральной кривой $\Gamma$ вещественны: $\lambda_{i} \in \mathbb{R}, i=1, \ldots, N, N<\infty$. Актуальной задачей является перенос этих результатов на случай кривых с комплексными точками ветвления, поскольку именно эта ситуация реализуется для большей части интегрируемых моделей. Эта проблема обсуждалась в ряде работ (см., например, [3], [4]), однако используемые в этих работах эвристические соображения, неудовлетворительны с математической точки зрения.

В этой работе мы демонстрируем характер возникающих трудностей и способы их преодоления на примере комплексных конечнозонных для физически важных моделей - нелинейного уравнения Шредингера (НШ)

$$
i u_{t}+u_{x x}+2|u|^{2} u=0,
$$

и модели Синус-Гордон (СГ)

$$
u_{x t}=\sin u \text {. }
$$

Именно эти модели обсуждались в работах [3], [4]. Используемая нами техника является модификацией методики [2] и основана на элементарном комплексном анализе.

Работа выполнена при частичной поддержке Российского фонда фундаментальных исследований, грант № 94-01-00193. 
Автор благодарен рецензенту за критические замечания, существенно улучшившие качество статьи.

\section{§ 1. Модель НШ}

Рассмотрим гиперэллиптическую риманову поверхность Г конечного рода $g>0$, заданную уравнением

$$
\begin{gathered}
z^{2}(\lambda)=\prod_{j=1}^{2 g+2}\left(\lambda-\lambda_{j}\right), \quad \lambda_{i} \neq \lambda_{j}, \\
\lambda_{j} \in \mathbb{C}, \quad j=1, \ldots, 2 g+2 .
\end{gathered}
$$

Точка $P$ на кривой Г задается парой $P=(\lambda, z)$. Мы будем иметь в виду обычное представление Г в виде двулистного накрытия комплексной плоскости $\mathbb{C}$. Выберем на Г канонический базис циклов $\left(a_{1}, \ldots, a_{g}, b_{1}, \ldots, b_{g}\right)$ с матрицей пересечений $a_{i} \circ a_{j}=b_{i} \circ b_{j}=0, a_{i} \circ b_{j}=\delta_{i j}, i=1, \ldots, g$; $j=1, \ldots, g$.

Пусть $\infty^{+}$и $\infty^{-}-$две бесконечно удаленные точки на $\Gamma: \lambda\left(\infty_{\stackrel{+}{-}}^{-}\right)=\infty$, $\sigma \infty^{+}=\infty^{-}$, где $\sigma$ - гиперэллиптическая инволюция на кривой $\Gamma$, именно, $\sigma:(\lambda, z) \rightarrow(\lambda,-z)$. Определим стандартные в теории конечнозонных решений модели НШ абелевы интегралы $\Omega_{1}(P)$ и $\Omega_{2}(P)$, имеющие асимптотики

$$
\begin{aligned}
& \Omega_{1}(P)=\mp\left(\lambda+\alpha_{1}+O\left(\frac{1}{\lambda}\right)\right), \quad P \rightarrow \infty^{ \pm}, \\
& \Omega_{2}(P)=\mp\left(\lambda^{2}+\alpha_{2}+O\left(\frac{1}{\lambda}\right)\right), \quad P \rightarrow \infty^{ \pm},
\end{aligned}
$$

и нормированные условиями

$$
\oint_{a_{i}} d \Omega_{j}=0 ; \quad i=1, \ldots, g ; \quad j=1,2 .
$$

Обозначим через $V^{(1)}$ и $V^{(2)} g$-мерные векторы $b$-периодов этих абелевых интегралов

$$
\begin{gathered}
V_{j}^{(1)}=\oint_{b_{j}} d \Omega_{1}(P), \quad V_{j}^{(2)}=\oint_{b_{j}} d \Omega_{2}, \\
j=1, \ldots, g .
\end{gathered}
$$

Справедливо следующее утверждение, усиливающее результат работы [3].

Tеорема 1. 1) Oтобрахсение $F: \mathbb{C}^{2 g+2} \rightarrow \mathbb{C}^{2 g+2}$

$$
\left(\lambda_{1}, \ldots, \lambda_{2 g+2}\right) \stackrel{F}{\rightarrow}\left(V^{(1)}, V^{(2)}, \alpha_{1}, \alpha_{2}\right)
$$


невырождено почти всюду. 2) В частности, это отображсение невырождено, если нули дифференциала $d \Omega_{1}(P)$ не совпадают с нулями дифференииала $d \Omega_{2}(P)$.

ДоКАЗАТЕЛЬСТво. Очевидно, что из пункта 2) теоремы 1 и аналитичности отображения $F$ следует утверждение пункта 1$)$. Для доказательства пункта 2) будем рассуждать "от противного".

Предположим $F$ вырождено, т.е. существует такая гладкая деформация $\Gamma(\tau), \tau \ll 1$, исходной кривой $\Gamma=\Gamma(0)$, что в то время, как левая часть (7) изменяется на $O(\tau)$, правая часть в $(7)$ изменяется на $O\left(\tau^{2}\right)$. Это утверждение в дальнейшем будем назьвать предположением.

Покажем, что сделанное предположение приводит к противоречию. Рассмотрим на кривой Г следуюшие объекты:

$$
v_{j}(P):=\left.\frac{\partial}{\partial \tau} \Omega_{j}(P, \tau)\right|_{\tau=0}, \quad j=1,2 .
$$

Здесь через $\Omega_{j}(P, \tau)$ обозначены абелевы интегралы, определенные с помощью (4) и (5), на деформированной римановой поверхности $\Gamma$.

Лемма 1. $v_{j}(P), j=1,2$ - суть мероморфные однозначные функчии на Г. Их особенности могут иметь лишь вид простых полюсов, расположенных в тех точках ветвления $\lambda_{k}$, где

$$
\left.\frac{\partial}{\partial \tau} \lambda_{k}\right|_{\tau=0} \neq 0
$$

ДоКАЗАТЕЛЬСТВо. Из определения (8) легко видеть, что $v_{j}(P)$ локально, в окрестности любой точки $P \in \Gamma$, являются мероморфными функциями на $\Gamma$. Особенности при $P \rightarrow \infty^{ \pm}$отсутствуют: в силу асимптотик (4) и нашего предположения о деформации имеем

$$
v_{j}\left(\infty^{ \pm}\right)=0, \quad j=1,2 .
$$

Покажем, что эти функции глобально определены на $\Gamma$, т.е. докажем их однозначность на $Г$. Действительно, в силу нормировок (6) $a$-периоды этих функций равны нулю. Согласно нашему предположению $b$-периоды также равны нулю. Это и означает, что $v_{j}(P)$ - корректно определенные на $Г$ мероморфные функции. Из определения этих функций (8) нетрудно извлечь, что вне точек ветвления $\lambda_{k}$ эти функции голоморфны. В окрестности точек ветвления имеем

$$
\left.v_{j}(P) \sim c_{k, j} \frac{\partial}{\partial \tau} \lambda_{k}\right|_{\tau=0} \frac{1}{\sqrt{\lambda-\lambda_{k}}},
$$

где $k=1, \ldots, 2 g+2, j=1,2$, а коэффициенты $c_{k, j}$ обращаются в нуль тогда и только тогда, когда $d \Omega_{j}\left(\lambda_{k}\right)=0$. Формула (9) описьвает структуру особенностей функций $v_{j}(P), j=1,2$. Лемма 1 доказана. 
ЛЕмма 2. Имеют место следующие представления

$$
\begin{aligned}
d \Omega_{1}(P) & =\frac{A(\lambda)}{z(\lambda)} d \lambda, & d \Omega_{2}(P) & =\frac{B(\lambda)}{z(\lambda)} d \lambda, \\
v_{1}(P) & =\frac{\widehat{A}(\lambda)}{z(\lambda)}, & v_{2}(P) & =\frac{\widehat{B}(\lambda)}{z(\lambda)},
\end{aligned}
$$

Здесь $A, B, \widehat{A}, \widehat{B}$ - полиномы от $\lambda$, причем

$$
\begin{array}{ll}
\operatorname{deg} A(\lambda)=g+1, & \operatorname{deg} B(\lambda)=g+2, \\
\operatorname{deg} \widehat{A}(\lambda) \leqslant g, & \operatorname{deg} \widehat{B}(\lambda) \leqslant g .
\end{array}
$$

ДокАЗАТЕЛЬСтво. Формулы (10) и (12) общеизвестны и легко вьводятся из теоремы Лиувилля, гиперэллиптичности римановой поверхности Г и асимптотик (4). Формулы (11), (13) выводятся аналогично с учетом леммы 1 и зануления функций $v_{j}(P), j=1,2$ при $\lambda \rightarrow \infty$. Лемма 2 доказана.

Лемма 3. Имеет место тождество

$$
\frac{v_{2}(P)}{v_{1}(P)}=\frac{d \Omega_{2}(P)}{d \Omega_{1}(P)} \quad \forall P \in \Gamma .
$$

ДокАЗАТЕЛЬСТво. Рассмотрим на Г следующий объект

$$
\dot{\Omega}_{2}(P)=\left.\frac{d}{d \tau} \Omega_{2}(\widetilde{P}(P, \tau), \tau)\right|_{\tau=0},
$$

где точка $\widetilde{P}(P, \tau) \in \Gamma(\tau)$ определяется из уравнения

$$
\begin{gathered}
\Omega_{1}(\widetilde{P}, \tau)=\Omega_{1}(P, \tau), \\
P \in \Gamma(0), \quad \widetilde{P} \in \Gamma(\tau) .
\end{gathered}
$$

Это уравнение однозначно разрешимо относительно $\widetilde{P}=\widetilde{P}(P, \tau)$ для почти всех $P \in \Gamma(0)$. Это следует из теоремы о неявной функции. Действительно, проблемы с разрешимостью уравнения (16) могут возникнуть лишь в $2 g+2$ точках на $\Gamma$, совпадающих с нулями дифференциала $d \Omega_{1}(P)$. Исследуем поведение функции $\dot{\Omega}_{2}(P)$ в этих точках. Из $(12),(13)$ легко вывести формулу

$$
\dot{\Omega}_{2}(P)=v_{2}(P)-v_{1}(P) \frac{d \Omega_{2}}{d \Omega_{1}}(P),
$$


из которой, в частности, следует, что $\dot{\Omega}_{2}(P)$ может иметь в нулях $d \Omega_{1}(P)$ особенности типа полюсов.

Однако, на самом деле, никаких особенностей там нет, поскольку

$$
\dot{\Omega}_{2}(P) \equiv 0 \quad \forall P \in \Gamma
$$

Действительно, из (17) следует, что $\dot{\Omega}_{2}(P)$ - мероморфная функция на $Г$. Подсчитаем ее нули и полюса. Покажем, что

$$
\dot{\Omega}_{2}\left(\lambda_{k}\right)=0, \quad k=1, \ldots, 2 g+2 .
$$

Этот факт проще всего увидеть из формулы (17) с учетом того, что $\dot{\Omega}_{2}(\sigma P)=-\dot{\Omega}_{2}(P)$, а члены порядка $O\left(1 / \sqrt{\lambda-\lambda_{k}}\right)$ в правой части $(17)$ сокращаются. Кроме того, функция $\dot{\Omega}_{2}(P)$ имеет нули в бесконечно удаленных точках $P=\infty^{(-+}$. Действительно, точки $\widetilde{P}\left(\infty^{(-)}, \tau\right)$, вычисляемые из уравнения (16), совпадают с бесконечно удаленными точками на $\Gamma(\tau)$ :

$$
\widetilde{P}\left(\infty^{ \pm}, \tau\right)=(\infty, \pm) \in \Gamma(\tau)
$$

Это значит, что они не зависят от $\tau$, и при вычислении $\dot{\Omega}_{2}\left(\infty^{\stackrel{+}{-})}\right)$ мы не должны учитьвать в $(15)$ зависимость от $\tau$ посредством $\widetilde{P}\left(\infty^{\stackrel{+}{-})}, \tau\right)$. Иными словами,

$$
\dot{\Omega}_{2}\left(\infty_{(-)}^{+}\right)=v_{2}\left(\infty_{(-)}^{+}\right)=0 .
$$

Итак, мероморфная функция $\dot{\Omega}_{2}(P)$ имеет по крайней мере $(2 g+4)$ нуля и не более, чем $(2 g+2)$ полюса. Это значит, что эта функция тождественно равна нулю. Лемма 3 доказана.

ЗАМЕчАниЕ 1. Наличие нулей в бесконечно удаленных точках (20) у функции $\dot{\Omega}_{2}(P)$ не удается усмотреть из представления (17). Действительно, на первьй взгляд кажется, что в этой формуле нуль функции $v_{1}(P)$ гасится полюсом функции $\left(d \Omega_{2} / d \Omega_{1}\right)(P), P \rightarrow \infty^{ \pm}$. Это отличает нашу ситуацию от модели Кд $\Phi$ (см [2]), а также СГ, где “дополнительньй" нуль в бесконечно удаленной точке можно было найти из представления типа (17).

ЛЕмма 4. В условиях теоремы 1 справедливы тожсдества

$$
v_{1}(P)=v_{2}(P) \equiv 0 \quad \forall P \in \Gamma \text {. }
$$


ДоКАЗАТЕЛЬСТво. Из тождества (14) и формул (10), (11) следует, что функция $v_{2}(P)$ имеет на Г нули более, чем в $2(g+2)$ точках, совпадающих с нулями $d \Omega_{2}(P)$ и $v_{1}(P)$. Здесь использовано то обстоятельство, что нули $d \Omega_{2}(P)$ и $d \Omega_{1}(P)$ не совпадают. С другой стороны, согласно (11) и (13), число нулей функции не превьшает $2 g$. Противоречие. Следовательно, $v_{2}(P) \equiv 0$, что, с учетом $(14)$, влечет $v_{1}(P) \equiv 0$. Лемма 4 доказана.

ЛЕмма 5. Деформачия спектральной кривой Г тривиальна:

$$
\left.\frac{\partial}{\partial \tau} \lambda_{k}\right|_{\tau=0}=0, \quad k=1, \ldots, 2 g+2 .
$$

ДОКАЗАТЕЛЬСТВо. Из Леммы 4 и того обстоятельства, что в локальном представлении (9) хотя бы один из коэффициентов $c_{k, j}$ не равен нулю (что является следствием условия теоремы 2 о несовпадении нулей дифференциалов $d \Omega_{1}(P)$ и $\left.d \Omega_{2}(P)\right)$ немедленно следует искомое равенство (22). Лемма 5 доказана.

Таким образом, мы пришли к противоречию с нашим исходным Предположением о нетривиальности деформации $\Gamma(\tau)$. Теорема 1 доказана полностью.

\section{§2. Модель Синус-Гордон}

В этом разделе мы используем старые обозначения для стандартных объектов конечнозначной теории СГ (2). Спектральную кривую будем по-прежнему обозначать через $\Gamma$, пару абелевых интегралов $\Omega_{1}(P)$ и $\Omega_{2}(P)$ и т.п.

Мы делаем это, чтобы не загромождать суть дела новыми обозначениями и сделать аналогию с $\S 1$ предельно ясной.

Несингулярная гиперэллиптическая римановаповерхность $\Gamma$ рода $g>0$ задается уравнением

$$
z^{2}(\lambda)=\lambda \prod_{j=1}^{2 g}\left(\lambda-\lambda_{i}\right), \quad \lambda_{i} \neq \lambda_{j},
$$

где точки ветвления $\lambda_{i}$ комплексны. Пусть $\Gamma^{+}-$верхний лист кривой $\Gamma$, а $\Gamma^{-}=\sigma \Gamma^{+}-$ее нижний лист. Выберем канонический базис циклов на $\Gamma\left(a_{1}, \ldots, a_{g}, b_{1}, \ldots, b_{g}\right)$ и определим абелевы интегралы $\Omega_{1}(P)$ и $\Omega_{2}(P)$, нормированные условием зануления $a$-периодов $(5)$ и фиксированные асимптотиками в точках $\lambda=\infty$ и $\lambda=0$, являющихся точками ветвления кривой $\Gamma$.

$$
\begin{array}{ll}
\Omega_{1}(P) \rightarrow \sqrt{\lambda}+O\left(\frac{1}{\sqrt{\lambda}}\right), & \lambda \rightarrow \infty, \\
\Omega_{2}(P) \rightarrow \frac{1}{\sqrt{\lambda}}+O(\sqrt{\lambda}), & \lambda \rightarrow 0 .
\end{array}
$$


Пусть $V^{(1)}$ и $V^{(2)}$ - векторы $b$-периодов интегралов $\Omega_{1}(P)$ и $\Omega_{2}(P)$ соответственно. Имеет место аналог теоремы 1 , описьваюший “частотное" отображение для модели СГ.

Tеорема 2. Отображсение $\Phi: \mathbb{C}^{2 g} \rightarrow \mathbb{C}^{2 g}$ вида

$$
\left(\lambda_{1}, \ldots, \lambda_{2 g}\right) \stackrel{\Phi}{\rightarrow}\left(V^{(1)}, V^{(2)}\right)
$$

обладает следующими свойствами невырожденности.

1) $\Phi$ невырождено всюду для $g=1$.

2) $\Phi$ невырохдено почти всюду для $g>1$.

3) В случае $g>1$ для невырожденности $\Phi$ достаточно, чтобы число общих нулей дифференциалов $d \Omega_{1}(P)$ и $d \Omega_{2}(P)$, лежсащих на верхнем листе $\Gamma^{+}$и не совпадающих с точками ветвления, было меньие двух.

ЗАМЕЧАНИЕ 2. Пункт 2), очевидно, является следствием пункта 3) и аналитичности отображения $\Phi$.

Аналогично (10) дифференциалы $d \Omega_{1}(P)$ и $d \Omega_{2}(P)$ могут быть записаны в виде

$$
d \Omega_{1}(P)=\frac{A_{g}(\lambda)}{z(\lambda)} d \lambda, \quad d \Omega_{2}(P)=\frac{B_{g}(\lambda)}{z(\lambda)} d \lambda,
$$

где $A_{g}(\lambda)$ и $B_{g}(\lambda)$ - полиномы степени $g$, и кроме того, $\left.B_{g}(\lambda)\right|_{\lambda=0} \neq 0$. В терминах этих полиномов пункт 3) теоремы 1 формулируется так: Полином $A_{g}(\lambda)$ либо не имеет общих нулей с $B_{g}(\lambda)$, либо $A_{g}(\lambda)$ и $B_{g}(\lambda)$ имеют лишь один общий нуль, не совпадающий с точкой ветвления $\lambda=\lambda_{i}$, $i=1, \ldots, 2 g$.

ДОКАЗАТЕЛЬСТВО ТЕОРЕМЫ 2. Основная линия рассуждений следует методике 1 . Приведем здесь лишь те фрагменты доказательства, которые отличаются от $\S 1$. Вновь предполагается, что $\Phi$ - вырожденное отображение и конструируются мероморфные функции $v_{1}(P)$ и $v_{2}(P)$, соответствуюшие абелевьм интегралам $(24)$. Легко убедиться, что функции $v_{j}(P), j=1,2$, допускают представление

$$
v_{1}(P)=\frac{\lambda \widehat{A}_{g-1}(\lambda)}{z(\lambda)}, \quad v_{2}(P)=\frac{\lambda \widehat{B}_{g-1}(\lambda)}{z(\lambda)},
$$

где $\widehat{A}_{g-1}(\lambda)$ и $\widehat{B}_{g-1}(\lambda)$ - полиномы по $\lambda$, степень которых не вьше $g-1$. Формулы (27) являются следствием представлений

$$
\begin{aligned}
& \Omega_{1}(P)=\int_{0}^{P} d \Omega_{1}(P), \\
& \Omega_{2}(P)=\int_{\infty}^{P} d \Omega_{2}(P)
\end{aligned}
$$


для абелевых интегралов $\Omega_{j}(P), j=1,2$, а также того факта, что точки ветвления $\lambda=0$ и $\lambda=\infty$ не зависят от $\tau$.

Лемма 6. Имеет место тождество

$$
\lambda A_{g}(\lambda) \widehat{B}_{g-1}(\lambda) \equiv \widehat{A}_{g-1}(\lambda) B_{g}(\lambda) \quad \forall \lambda .
$$

ДокаЗАТЕЛЬСтво. Тем же способом, что и в лемме 3 , устанавливается тождество (14). (При этом для доказательства вспомогательного тождества $\dot{\Omega}_{2}(P) \equiv 0$ можно пользоваться как определением (15), так и представлением (17).) Сравнивая (14), (26) и (27), приходим к искомому тождеству (28). Лемма 6 доказана.

Из леммы 6 немедленно следуют утверждения:

a) $\widehat{A}_{g-1}(0)=0$;

б) $\operatorname{deg} \widehat{B}_{g-1}(\lambda) \leqslant g-2$;

в) полином $A_{g}(\lambda)$ имеет не менее двух обших нулей с полиномом $B_{g}(\lambda)$.

Очевидно, в условиях теоремы 2 последнее условие не выполнено. Следовательно, с необходимостью $\widehat{A}_{g-1}(\lambda)=\widehat{B}_{g-1}(\lambda) \equiv 0$, и должны быть выполнены тождества $v_{1}(P)=v_{2}(P) \equiv 0$.

Остается сделать последний шаг - вывести из этого тождества аналог леммы 5. Для $g>1$ это очевидным образом следует из нашего ограничения на нули $d \Omega_{1}(P)$ и $d \Omega_{2}(P)$. Итак, $d \lambda_{i} /\left.d \tau\right|_{\tau=0}=0, i=1, \ldots, 2 g$. В случае $g=1$ единственный нетривиальньй случай, заслуживающий анализа - это попадание совместного нуля наших дифференциалов в точку ветвления $\lambda=\lambda_{i}$ :

$$
d \Omega_{1}\left(\lambda_{i}\right)=d \Omega_{2}\left(\lambda_{i}\right)=0 .
$$

Покажем, что это вариант невозможен. Пусть для определенности $\lambda_{i}=\lambda_{1}$.

Рассмотрим дифференциал $d \widetilde{\Omega}(P)=\xi d \Omega_{1}(P)+d \Omega_{2}(P)$, где константа $\xi$ выбрана так, что $d \widetilde{\Omega}(P)$ имеет в точке $\lambda=\lambda_{1}$ нуль второго порядка.

Рассмотрим линии уровни для мнимой части абелева интеграла $\widetilde{\Omega}(P)$ :

$$
L:=\left\{\lambda \in \mathbb{C}: \nu(P)=\operatorname{Im} \int_{\lambda_{1}}^{P} d \widetilde{\Omega}(P)=0\right\}
$$

и рассмотрим локальную картину множеств $L$ вблизи особых точек $\left\{\lambda_{1}\right.$, $\left.\lambda_{2}, 0, \infty\right\}$. Из точки $\lambda=\lambda_{1}$ выходит пять "усов", принадлежащих $L$. А из точек $\lambda=\lambda_{2}, \lambda=0, \lambda=\infty$ выходит по одному "усу". Очевидно, два уса, выходящих из точки $\lambda=\lambda_{1}$, замыкаются в петлю $Q$. Гармоническая функция $\nu(P)$ обрашается в нуль на границе $Q$, следовательно, по принципу максимума, $\nu(P) \equiv 0$ внутри $Q$. Но это противоречит явным формулам для дифференциала $d \widetilde{\Omega}(P), d \widetilde{\Omega}(P) \not \equiv 0$. Итак, свойство (29) невозможно 
для $g \geqslant 1$. Значит, либо $d \Omega_{1}\left(\lambda_{i}\right) \neq 0$, либо $d \Omega_{2}\left(\lambda_{i}\right) \neq 0$. Отсюда следует тривиальность деформации

$$
\left.\frac{d \lambda_{i}}{d \tau}\right|_{\tau=0}=0, \quad i=1,2, \ldots, 2 g, \quad g \geqslant 1 .
$$

Теорема полностью доказана.

ЗАмечАниЕ 3 . В случае, когда спектральная кривая $\Gamma$ допускает дополнительную антиинволюцию типа комплексного сопряжения $\pi: \lambda \rightarrow \bar{\lambda}$, $z \rightarrow \bar{z}$ (физически интересньй случай модели СГ) разбор специального случая $g=1$ сильно упрошается: невозможность равенства (29) сразу следует из соображений симметрии.

Институт математики и механики

Поступило

Уральского отделения РАН

29.11 .93

\section{СПИСОК ЦИТИРОВАННОЙ ЛИТЕРАТУРЫ}

[1] Krichever I. M. Perturbation theory in periodic problems for two-dimensional integrable systems // Sov. Sci. Rev. C. Math. Phys. 1991. V. 9. P. 1-101.

[2] Bikbaev R.F., Kuksin S. B. On the parametrization of finite-gap solutions by the frequency and wave number vectors and a Theorem of I. Krichever // Lett. Math. Phys. 1993. V. 28. P. 115-122.

[3] Бикбаев Р. Ф.Алгебро-геометрические неравенства, порождаемые теорией возмущений: нелинейные уравнения Шредингера // Алгебра и анализ. 1993. T. 5. № 4. C. $67-82$.

[4] Бикбаев Р. Ф., Куксин С. Б. Периодическая краевая задача для уравнения Синус-Гордон, ее малые гамильтоновы возмущения и КАМ-деформации конечнозонных торов // Алгебра и анализ. 1992. Т. 4. № 3. С. 42-78. 